\title{
Under Review: Data Requirements and Method Development of a New Bee Risk Assessment Scheme for Plant Protection Product Registration
}

\author{
Lukas Jeker* and Daniela Grossar
}

\begin{abstract}
An up-to-date ecotoxicological risk assessment of plant protection products (PPPs) depends on the constant improvement of risk assessment methods and guidelines, and a thorough evaluation of their impacts. Here, we explain how the risk assessment of PPPs with regard to bees and the authorisation of PPPs is conducted in Switzerland. We further report the design and application of a new method to study homing flights of honey bees using the Radio Frequency Identification (RFID) technique. The new method allowed to address the effects of sublethal doses of two neonicotinoids, thiamethoxam and thiacloprid, on the flight capacities of honey bees. Currently, this study design is under evaluation in an international ring test, in which the Swiss Bee Research centre participates. It is the first test design focussing on sublethal effects of PPPs on honey bees and a draft method will be submitted to OECD to become an official test guideline in the near future. Potential shortcomings and ideas for refinements on the RFID test design are discussed.
\end{abstract}

Keywords: Honey bee $\cdot$ Plant protection product authorization $\cdot$ RFID $\cdot$ Swiss national action plan

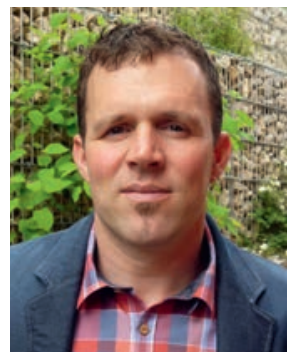

For the last 25 years Lukas Jeker has worked as an agricultural biology laboratory assistant, study director and team leader in the field of terrestrial ecotoxicology in various companies and contract research organisations (CRO) in Switzerland and England. His tasks included the performance, development and risk assessment of regulatory studies with non-target arthropods specializing in honey bees for plant protection product (PPP) registration. At Agroscope he is working in the Swiss Bee Research Centre (SBRC) as a scientific collaborator. His tasks include the scientific evaluation of the risks of plant protection products to bees, the preparation of expert opinions for the Federal Office for Agriculture's (FOAG) licensing office within the scope of licensing in Switzerland, the validation and development of international test methods for the evaluation of the risks of PPPs for bees (OECD, EFSA, ICPPR and SETAC) as well as advising the FOAG on questions of bee toxicology.

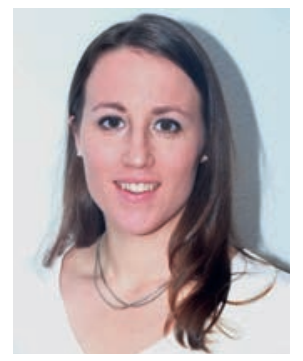

After completing her Master's degree in zoology with focus on honey bee biology at Karl-Franzens University in Graz (Austria), Daniela Grossar came to Bern in 2012 to start a $\mathrm{PhD}$ project at Agroscope's Swiss Bee Research Centre (SBRC). During her studies she gained experience in the risk assessment of plant protection products to bees on the one hand as an assistant in field studies, on the other hand she was involved in the deskbased evaluation of data for PPP authorization in Switzerland. In June 2019, she started working as scientific collaborator at the SBRC for the risk assessment of plant protection products to bees.
Plant protection products (PPPs) play a major role in controlling pests that endanger agricultural plant production to feed a rapidly growing world population. The use of PPPs increased approximately 50 -fold in the 20 th century ${ }^{[1]}$ and this trend may even intensify in the next decades, as the world's population is expected

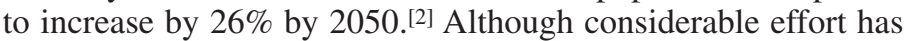
been directed toward the adoption of holistic, non-chemical pest control management strategies, the integration of such presumably successful methods in nation- or region-wide scale appears to be lengthy and cost-intensive. ${ }^{[3]}$ The sustainable and responsible use of agrochemical substances (PPPs and fertilizers) enhancing agricultural productivity, is therefore a reliable interim solution to prevent food shortages.

About $35 \%$ of the global food supply depends on the pollination of crops by animals, most of them being insects. ${ }^{[4]}$ Animal pollination is an indispensable ecosystem service, provided particularly by bees, hoverflies and butterflies. ${ }^{[5]}$ Among them, honey bees are considered to be the most important pollinators of both cultivated and wild plants. Recent studies indicate that the role of wild bees in plant and crop pollination service is significantly underestimated. ${ }^{[6]}$ Wild bees seem to be equal, more efficient or even the sole pollinators of certain flowering plants. However, wild bees are often strongly bound to small local niches, highly specialized to only a very few types of plants, or only appear in small numbers on a community wide scale compared to honey bees, which are generalists, exploiting a wide range of flowers for nectar and pollen. Premarketing PPP risk assessment schemes therefore have to include testing for acute and sublethal effects of PPPs on domesticated and wild pollinators.

Apart from the direct benefits of PPP use brought to humans by protecting crops from pests, PPPs and their metabolites can have indirect adverse effects on human health (PPPs' user or food consumer) and the environment. The safe use of PPPs, thus, ap- 
pears as one of the biggest challenges of agricultural intensification in the $21^{\text {st }}$ century. In this area of conflict, we have to agree on a reasonable compromise between protection, as attempted by conservation of biodiversity and especially the protection of beneficial organisms including bees, and concurrently being able to feed a growing world population. Improving PPP policy and regulation may be a valuable option mediating between these sometimes conflicting objectives.

\section{Plant Protection Product Registration in Switzerland}

Authorization of a new plant protection product (PPP) is granted only if the proposed uses of the PPPs are expected not to have unacceptable adverse effects on the environment, on animal or on human health, and is regulated by the Swiss Ordinance on Plant Protection Products (SR 916.161[7]). After submission of a registration dossier to the Federal Office for Agriculture (FOAG) by an applicant, usually in the person of the manufacturing company or of a PPP distributor, the FOAG supervises the submitted data for its completeness regarding the following information:

- identity of active substance(s) of the product and efficacy of the PPP,

- human toxicity and toxicology in mammals,

- metabolic pathway and residues in plants, crops and livestock,

- environmental fate and behaviour in soil, water and air and

- ecotoxicological impact on several soil-, aquatic- and terrestrial non-target organisms.

The FOAG administers the authorization process and distributes the file or dossier to expert groups for further assessments of the information provided. One of these expert groups is based at the Swiss Bee Research Centre (SBRC), which is part of Agroscope, a public research institute for Agriculture, and monitors and assesses the potential effects of PPPs on bees, i.e. honey bees, bumble bees and solitary bees.

A PPP may only be authorised if an appropriate risk assessment was conducted, which ensures that effects on adult bees and larvae, their behaviour or survival, or on the development of bee colonies are negligible. Data requirements for the registration of PPP in Switzerland are based on the regulation of the European Parliament and the Council (Regulation (EC) No 1107/2009[8]) including the regulation (EU) No 283/2013[9] setting out the data requirements for active substances and regulation (EU) No $284 / 2013^{[10]}$ for formulated plant protection products, respectively. ${ }^{[11]}$ As described in an extensive guidance document on the risk assessment of PPPs on bees by the European and Mediterranean Plant Protection Organization (EPPO) ${ }^{[12]}$ and as suggested by the European Food Safety Authority (EFSA) ${ }^{[13]}$ a tiered risk assessment scheme with a stepwise approach from laboratory (lower tier) to semi-field to field (higher tier) studies is pursued in Switzerland and the EU. Lower tier testing comprises of laboratory-based acute toxicity (OECD 213[14] and OECD 214[15]) studies on adult bees, where median lethal doses (LD50 value for oral and contact exposure in $\mu \mathrm{g} / \mathrm{bee}$ ) of the active substance or of a PPP itself are determined. Oral chronic exposure assays on adult bees (OECD 245[16]) and on bee larvae (OECD 237[17] and OECD GD $239[18])$ are required for the active substance only, to determine the toxicity of the chronic oral intake of active substances entering the bee hive via contaminated pollen and/or nectar. If increased mortality of adult bees and/or larvae after exposure in these first tier tests is noticed or sublethal effects on growth or development of bees cannot be excluded, higher tier testing is required to complement these initial experiments.

Sublethal effects summarize all adverse effects of an active substance to bees that impair but do not lead to the immediate death of an individual bee or bee colony, such as the development of hypopharyngeal glands in adult nurse bees, necessary for producing a secrete fed to the bee larvae, or the orientation and memory ability of forager bees, which can be tested via the new RFID test method (see below). Higher tier studies are conducted under semi-field or field conditions (EPPO 170[12a]) and in which queen-right bee colonies are confined in tunnels for a limited exposure time with flowering plant cultures treated with the PPP to be tested or set up next to a treated crop under real field conditions, respectively. In comparison with laboratory tests, semi-field experiments permit the simulation of more realistic conditions and combine advantages of laboratory tests, e.g. controlled exposure of bees with the PPP, with the advantages of field studies, e.g. including the natural variability and the complex interactions within a bee colony and the bee colony with the environment (e.g. weather conditions, food availability). At the same time, semi-field experiments avoid disadvantages, as an oversimplified approach dealing with worst cast scenarios as in the laboratory-based studies, or the extensiveness in manpower and costs in field studies. However, both types of higher tier risk assessment studies allow for the analysis of parameters relevant for full bee colonies, such as colony and brood development (Oomen brood test ${ }^{[19]}$ and OECD $75^{[20]}$ ), foraging activity, adverse effects on bee health and vitality, or overwintering success, which are not feasible in lower tier studies. All these studies necessary for authorization of PPPs are conducted according to internationally recognized methods established by the Organisation for Economic Cooperation and Development (OECD) or the Plant Protection Organisation for Europe and the Mediterranean (EPPO). Since 2018, bumble bees (Bombus terrestris L.) (OECD 246[21] and OECD 247[22]), as representatives for a large and diverse group of more than 580 wild bee species occurring in Switzerland [23] are included into the risk assessment of PPPs on bees. Furthermore, new methods for bumble bees (Bombus terrestris L.) and the solitary red mason bees (Osmia bicornis L.) are being developed and currently under validation via international ring testing setups. PPP manufacturers commonly commission autonomous and independent contract research organizations (CROs) with the execution of PPP regulatory studies according to good laboratory practices (GLP).

In Switzerland, the study reports on the ecotoxicological risk of a PPP to bees are collated in the registration dossier submitted to the FOAG by which the data is assigned to the PPP bee risk assessment group, which is part of the SBRC (Agroscope). Risk assessment by the experts of the SBRC involves considering the possibility or the expected exposure of bees by calculating the concentration of the PPP in the field and relating it to bee toxicity based on the available data. In the current risk assessment, the hazard quotient (HQ), is determined by dividing the application rate $(\mathrm{g} / \mathrm{ha})$ of a PPP or its active substance by the bee LD50 oral/contact value ( $\mu \mathrm{g} / \mathrm{bee})$ of the acute toxicity laboratory study. Additionally, the toxicity exposure ratio (TER) is calculated by the ratio of the bee toxicity, determined in chronic oral exposure assays, to the potentially worst-case exposure level (e.g. residue levels of a PPP in nectar and pollen). Lower tier tests are employed as first tier risk indicator for the spray application of a PPP to bees. From HQ values below 50 and/or TER values above one, a low risk to bees is concluded. A HQ value exceeding 50 and/or a TER value below one mostly triggers the realisation of higher tier studies to prove an acceptable risk under more realistic (semi-field) or realistic (field) exposure conditions.

If not, these data are used for setting up mitigation measures of the use of the PPPs in accordance of the risk assessment scheme and can result in specific use restrictions or ban. In the case the complete risk assessment of a PPP requires more data, the experts are entitled to claim more study results or the repetition of a study from the PPP registration applicant. For the final decision on the approval of a PPP in Switzerland, the FOAG consolidates the expertise from all expert groups, distributed over five federal offices and its subdivisions, including the Federal Office for the Environment (FOEN), the Federal Office of Public 
Health (FOPH), the Federal Food Safety and Veterinary Office (FSVO), the State Secretariat for Economic Affairs (SECO) and the Research Centre Agroscope affiliated to the FOAG. Whenever necessary, restrictions on the use of a product are imposed to avoid possible risks for bees. The risk management for the protection of bees is effectuated using defined application measures specified in legal obligations. Safety regulations concerning bee protection management start with the code 'SPe 8 - dangerous to bees' and are imprinted on the label, the package leaflet and/or container of a product. These safety instructions for the application of product classified as hazardous to bees are obligatory and intended to substantially reduce exposure of bees at an acceptable level, or ideally, completely avoid the contact with the PPP when application is carried out according to good agricultural practice (GAP). If there is a modification in the intended use of the PPP other than specified in the authorization, e.g. the usage of PPP is planned to be expanded to another plant culture, or the composition of the PPP changes, the holder of the PPP permit is obliged to report these changes at the FOAG and the registration process is restarted. However, PPPs are subjected to a continuous review, in particular if new studies that show adverse effects of the active ingredient on bees, non-target organisms, human health or the environment are published, these substances are reassessed by the concerned expert group and the FOAG for their risks.

\section{The Swiss National Action Plan for Bee Health and New Data Requirements for PPP Registration}

In 2014, the Swiss government installed a national action plan promoting bee health to counteract increasing honey bee colony losses in preceding years. The plan stipulates several measures to prevent the further loss of bee colonies and ensures the pollination service by bees in future, including: 1) the support of farmers with direct payments to incentivize the setup of flower strips on their farm land, as food and habitat resource for bees; 2) the funding of research targeting disease control in honey bees and conservation of honey and wild bee populations; 3 ) the formation of a bee health service organisation offering help to beekeepers and stakeholders, and improving beekeeping practice in Switzerland; 4) the implementation of buffer strips around PPP treated fields, where flowering or plants containing honeydew have to be removed before application of a PPP; and 5) the definition of new data requirements for the registration of a PPP.

In the course of the implementation of the Swiss national action plan for bee health, a revision of the risk assessment scheme for the authorization of PPPs in Switzerland took place. Due to the development of PPPs with new modes of action, new routes of PPP exposure to bees were identified and included into the risk assessment of PPPs to bees. New aspects are or will be evaluated: the risk posed to bees by metabolites of PPPs, the risk incurred to bees by the collection of contaminated water, the risk of chronic exposure to PPPs, the risk posed to wild bees (bumble bees and solitary bees) and the investigation of sublethal effects, such as effects on hypopharyngeal gland development, or effects on memory and the orientation of bees.

\section{The Development of a Test System for Assessing the Homing Flight Abilities of Bees after PPP Exposure}

The risk assessment of PPPs is constantly being refined and evolving, in order to attain the best possible protection of bees and other non-target organisms. Hence, there is a need for the development and validation of new testing methods before their integration into the current applicable risk assessment scheme. The data quality of PPP risk assessment studies to bees is of utmost importance, as a sound and reliable risk assessment, which has an impact on a possible approval of a PPP, is only feasible on the base of reliable data. Consequently, new testing methods undergo a validation process, commonly executed by an inter-laboratory test performed independently several times from independent scientist according to the same protocol, which analyses whether the efficiency and reliability of the new methods meets defined quality criteria. Since 2016, the SBRC participates in the international ring test for the validation of a RFID test method ${ }^{[24]}$ to assess the homing flight abilities of bees after PPP exposure.

The aim of the RFID homing flight ring test ${ }^{[24]}$ is to develop a method for assessing the sublethal effects of xenobiotic substances, e.g. active substances of PPPs, on the navigation of foraging bees (RFID ring-test protocol 2016-2019, unpublished; method similar to Decourtye et al. $\left.{ }^{[25]}\right)$. RFID is an automatic and contactless communication technology and serves for the identification e.g. of persons, animals and goods. A RFID system consists of a data carrier, called tag or chip, and a reader. The reader emits weak electromagnetic waves that are reflected by the tag and provide the reader with information about passing tags. The passing of tags is logged contactlessly and recorded on the reader's memory. This method is used to analyse the flight behaviour by the means of the return rate and return time of foraging honey bees back to the hive after exposure to a PPP. The inter-laboratory comparison is carried out in twelve laboratories of five countries: Germany, Italy, England, France and Switzerland.

\subsection{RFID Test Method}

A sufficient number of forager bees are caught in front of the hive entrance and marked in groups with a non-toxic colour powder. They are then brought to a release location one $\mathrm{km}$ away from their hive and released again. The bees familiar with the environment normally fly directly back to their hive. The returning bees are clearly identifiable by the colour coding, they are collected again and prepared for the RFID test. This pre-selection is necessary to ensure that only foragers familiar with the environment are used for the test. The collected bees are then divided into groups of ten bees $(3 \times 10$ bees per treatment $)$ confined to a plastic cage. To identify the treatment, a passive RFID chip is glued with tooth cement dorsally onto the thorax of each bee (Fig. 1). Each bee group (ten bees per cage) receives $200 \mu \mathrm{L}$ of $30 \%$ (w/V) sucrose solution (control group), or the same amount of sucrose solution containing a defined sublethal concentration of a PPP (treatment groups). The distribution of the offered treatment solution among the caged bees takes place through trophallaxis, the zoological term for the transfer of liquid feed from one animal mouth to another developed mainly in social insects (e.g. honey bees, ants). It is assumed that the food distribution $(20 \mu \mathrm{L}$ per bee) by trophallaxis and thus the PPP dose per bee is the same among the bees confined in one cage. After complete consumption of the provided dosage, or after a maximum exposure time of $1.5 \mathrm{~h}$ and a subsequent starvation time of $0.5 \mathrm{~h}$, the exposed and treated bees are returned to the same release site as before, one $\mathrm{km}$ away from their hive, and released simultaneously. The entrance of the test bee hive is

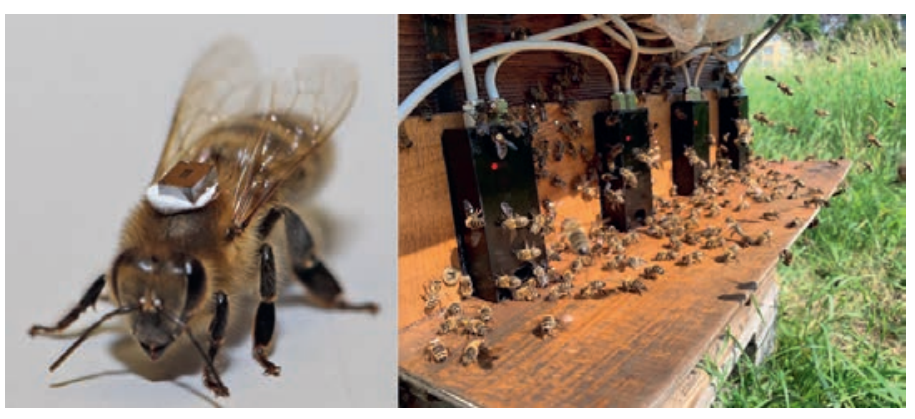

Fig. 1. Honey bee with Radio Frequency Identification (RFID) chip glued dorsally onto thorax (left). Image of the entrance with flight board of a test bee hive equipped with four RFID readers. Bees with RFID chips are recorded when passing the reader (on the right). 
equipped with four RFID readers (Fig. 1), hence all bees of the colony are forced to enter the hive using one of them. Within 24 $\mathrm{h}$, returning bees tagged with an RFID chip are identified and recorded each time they pass the reader and their return rate and return time is monitored. To evaluate the effects of PPPs on the flight abilities of honey bees the return rate and time of treated bees is statistically compared among treatment groups and the untreated control. The main variable return rate, as indicator for the homing flight success, is in binary format: 1 if the honeybee returns to the hive and passes the reader in the $24 \mathrm{~h}$ period, or 0 if not. The return time, measuring the time span between the release and the first recording of the bee at the hive is a quantitative variable, which is only recorded for bees that are able to return to their hive and also pass the RFID readers at the entrance. Each participating laboratory replicates the experiment thrice for each treatment on different bee colonies.

The RFID test method is still under evaluation, however, a draft test guideline will be submitted in early 2020 to OECD, in which the results of the ring test are compared and discussed. How the results of the RFID method will be included and handled in a risk assessment scheme for sublethal effects is still under discussion. An important question is whether the concentrations tested are field realistic, hence, if the active substance of a PPP is present in pollen and nectar of the treated plants after PPP application in amounts that might cause sublethal or toxic effects to bees. To answer this question, residue studies are required in which the concentration of an active substance is measured after a PPP application in the bee matrices pollen, nectar and honey. A further important question is how a potential influence on the return rate of the individually tested forager homing flight return rate and time among treatment groups and an untreated control, which received only $30 \%$ (w/V) aqueous sucrose solution. For both release sites the experiment was replicated three times. The mean homing flight returning rate at both sites was $94 \%$ with little deviation (79-100\%) and no statistically significant difference between landscapes and among treatments was observed (two-tailed Welch t-test on arcsine transformed return rates, with Bonferroni correction, $\mathrm{p}=0.172$ ). However, the duration of the homing flight of bees exposed to the highest dose $(1.0 \mathrm{ng} / \mathrm{bee})$ of thiamethoxam was statistically significantly higher compared to the others (Kruskal-Wallis-test with post-hoc Bonferroni correction for multiple pairwise comparisons, $\mathrm{p}<0.05$; Fig. 3 ). Homing flights of all treatments in the urban landscape were longer than performed in the rural landscape (Kruskal-Wallis test, post-hoc Bonferroni correction for multiple pairwise comparisons, $\mathrm{p}<0.05)$. Further, we tested interactions between treatment and landscape on the return rates and homing time of bees by an univariate generalized linear model: Treatment has an effect $(\mathrm{p}=0.010)$, landscape individually has no effect $(p=0.296)$ and there is no effect of the interaction on the return rate $(\mathrm{p}=0.769)$. The model applied on the homing time shows that treatment has an effect $(p<0.000)$, landscape has an effect $(p=0.002)$, but interaction of treatment $\times$ landscape had no effect on the return time $(p=0.545)$. Our data shows that landscape might have an impact on the cognitive orientation capacity, hence play a role in the duration of the homing flight. The urban landscape used in our study provides more obstacles than the rural landscape chosen here, which most likely resulted in a longer homing flight duration. Nevertheless, in both locations and in all treatments, the homing rates did not statistically significantly differ from each other underlining that sublethal doses were tested. This finding sup-

Fig. 2. Satellite pictures (www.geo admin.ch) showing the urban (left) and the rural (right) landscapes used for the RFID experiments. Bees challenged with thiamethoxam had to return from the release site $(A)$ to their colonies $(B)$ in one $\mathrm{km}$ distance.
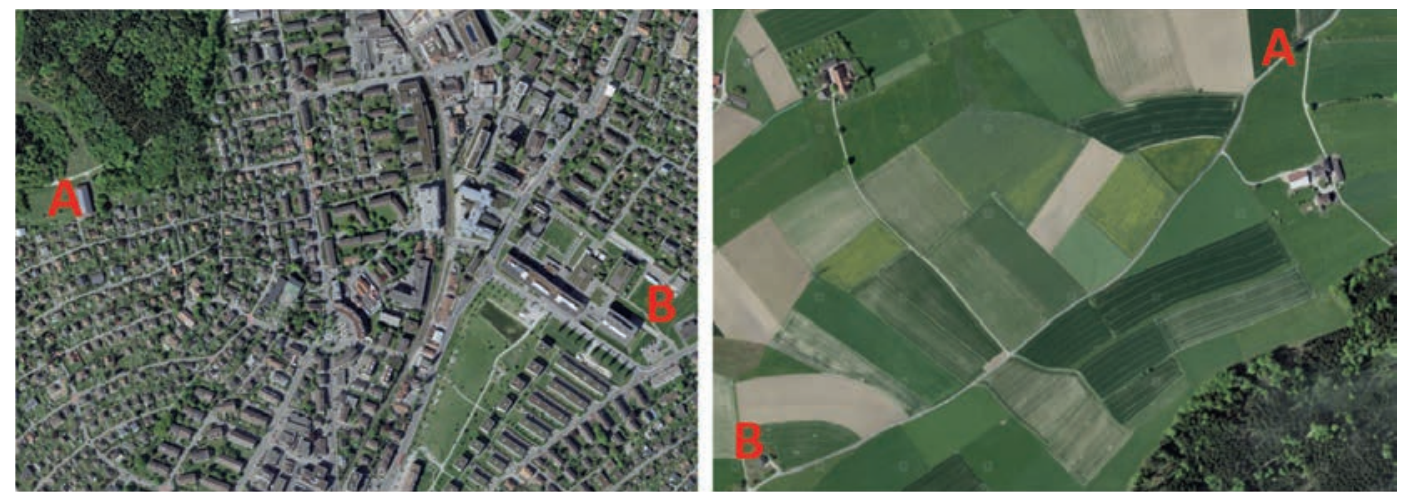

bees affects the honey bee colony ecosystem. Data on colony effects of PPPs help to assess potential hazards from sublethal doses of PPPs and allow to deduce appropriate safety measure for the application of a PPP.

\subsection{Approval of Additional Parameters, which might Influence the Outcome of the RFID Method}

The SBRC conducted additional experiments, in order to identify factors that could distort the results of the RFID ring test method.

I) Assuming that different landscape types influence the orientation ability of bees, and consequently might have an impact on the time bees need to fly back to their hive, we challenged the RFID test method by choosing two release sites for the test bees, where they had to encompass two contrasting landscapes. The first release site was situated in an urban environment, characterized by buildings and streets. The second release site was set in a rural area surrounded by farmland and less housing (Fig. 2). Forager bees (N $=30$ or 40 bees per treatment group) were orally exposed to the neonicotinoid thiamethoxam in sublethal doses of $0.1,0.3$ and 1.0 ng per bee following the RFID ring test protocol, and compared

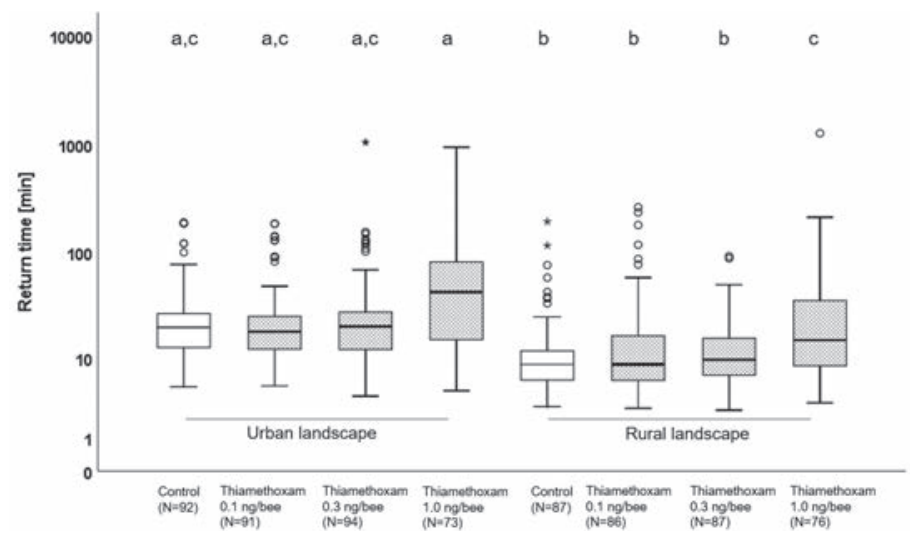

Fig. 3. Return times of honey bees after treatment with different doses of thiamethoxam in two different landscapes, urban (left) and rural (right), in the RFID homing test. The y-axis is depicted in a logarithmic scale as return time shows high variance and stars and circles depict outliers. Letters on top of boxplots mark statistical different groups (KruskalWallis test, two-sided, post-hoc Bonferroni correction for multiple pairwise comparisons, $\mathrm{p}<0.05)$. Similar letters on top indicate no statistical difference between the return times of treatment groups. 
ports the validity of the tested method when using treatment doses from 0.1 to $1.0 \mathrm{ng}$ thiamethoxam per bee, but does not exclude landscape/treatment interactions if thiamethoxam in higher doses or other substances are administered.

II) We further tested a possible bias on the results of the RFID method caused by a dosing effect due to an uneven syrup distribution among the ten bees of a treatment group. The food sharing via trophallaxis has been shown to be unequal among caged honey bees.[26] Therefore, we also suspected a non-uniform distribution of the administered sucrose solution among bees of the ten-bee group feeding regime as proposed in the RFID ring-test protocol. To address this issue, we compared two different feeding schemes in the RFID test by exposing honey bees caged in groups of two or ten orally to the sublethal concentration of $1.0 \mathrm{ng}$ thiamethoxam per bee, or control bees that received pure sucrose instead. Each treatment group consisted of 30 bees, either confined in cages with 10 bees each, or confined in fifteen cages with two bees per cage, and the experiment was replicated three times. For the analysis of vitellogenin gene expression, returning bees of the RFID homing test, including both feeding schemes, were collected and frozen at $-20{ }^{\circ} \mathrm{C}$ for subsequent molecular analysis. RNA isolation, cDNA synthesis and qPCR for vitellogenin gene expression analysis on honey bee brains was carried out following the protocol of Christen et al. ${ }^{[27]}$ Our results show that the mean homing flight success rate of honey bees, after treatment with thiamethoxam, was considerably lower in the group of ten bees (57\%), compared to the two-bee feeding approach (80\%), as well as to the controls (in both controls $88 \%$ mean return rate). However, a very large variability of homing success rate (36-79\%) was detected in the ten-bee feeding group, but not in the two-bee feeding approach (77-83\%; Fig. 4), and no statistically significant difference in the return rates among treatment groups was identified (Welch t-tests among treatment groups on arcsine transformed return rates, $\mathrm{p}=0.044$, and pairwise tests with post-hoc Bonferroni correction for multiple pairwise comparisons found no differences $\mathrm{p}>0.073$ ). Return time is varying strongly among treated and untreated bees (Fig. 5) and the median return time in bees treated with $1.0 \mathrm{ng}$ thiamethoxam is slightly higher (38.2 $\mathrm{min}$ and $16.3 \mathrm{~min}$ in ten- and two-bee feeding, respectively) than in untreated controls (14.0 min and $13.8 \mathrm{~min}$ ).

Vitellogenin is an important transcript regulating foraging activity, and vitellogenin expression is induced upon thiamethoxam exposure. ${ }^{[27]}$ Fold change of vitellogenin transcripts in our experiment differed among treatment groups (two-sided Kruskal-Wallis test, $\mathrm{p}=0.007$ ) and showed less variation in the two-bee feeding mode than in the ten-bee feeding scheme (Fig. 6). We found a statistically significantly higher expression of vitellogenin in

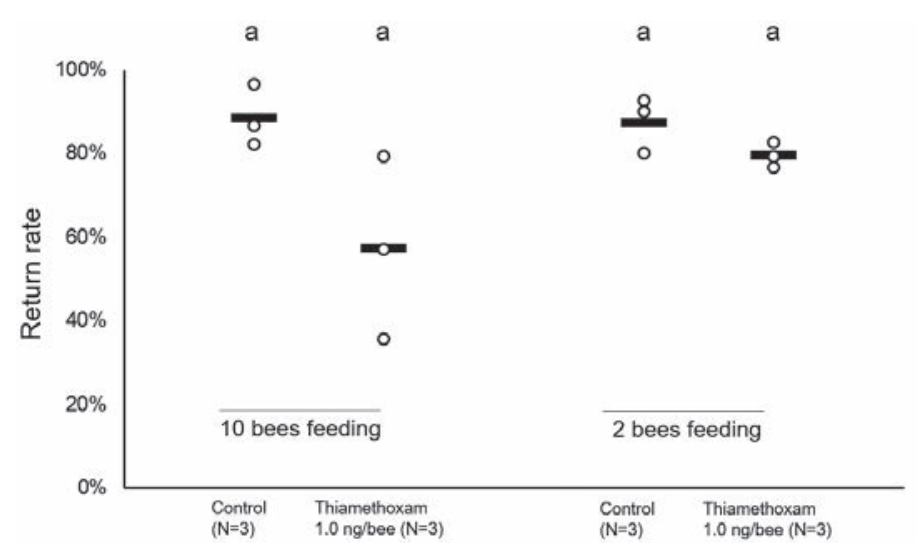

Fig. 4. Return rates of honey bees in the RFID homing test after treatment with $1.0 \mathrm{ng}$ thiamethoxam per bee in two distinct feeding schemes: bees treated with thiamethoxam in a ten-bee feeding mode (left) and bees treated in a two-bee feeding approach (right). Circles depict percentages of returning bees of three individual runs with 30 bees per treatment, lines represent the mean of these three runs. samples of the two-bee feeding scheme compared to the samples fed in the ten-bee group mode (pairwise Kruskal-Wallis tests with Bonferroni correction, $\mathrm{p}=0.041$ ) and to controls (pairwise Kruskal-Wallis tests with Bonferroni correction, $p=0.008$ and $p$ $=0.029$ ). The variance of both, the return rate in the RFID homing flight test and also vitellogenin expression, is substantially higher among bees belonging to the ten-bee feeding scheme than in bees of the two-bee feeding scheme. It can be suggested that this variance is caused by an unequal food sharing within larger groups of caged honey bees and was already demonstrated in Brodschneider et al., ${ }^{[26]}$ which potentially hampers the exact dosing of xenobiotic substances in ecotoxicological experiments on bees, and hence bias their outcome. Furthermore, it should be noted that only returning bees were used for vitellogenin expression analysis assuming that overdosed bees were absent (not returning), while the return rate evaluation included all treated bees. Thus, a possible treatment effect from the analysis of underdosed and overdosed returning bees is more pronounced than in the vitellogenin analysis, which probably consisted mainly of underdosed returning bees of the ten-bee feeding group. Our findings therefore emphasize the need for improvements in the RFID ring test method, as well as generally in exposure tests of PPPs to bees.

III) Foragers of a honey bee colony collect either nectar or pollen, and only a few of them gather both.[28] The type of food

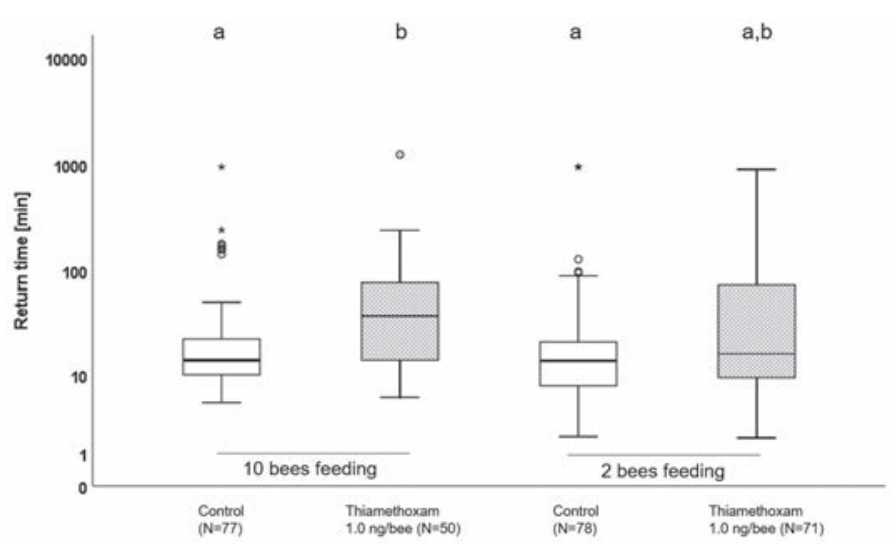

Fig. 5. Return times of honey bees in the RFID homing test after treatment with $1.0 \mathrm{ng}$ thiamethoxam per bee in two distinct feeding schemes: bees treated with thiamethoxam in a ten-bee feeding mode (left) and bees treated in a two-bee feeding approach (right). Letters on top of boxplots mark statistical different groups (Kruskal-Wallis test, two-sided, post-hoc Bonferroni correction for multiple pairwise comparisons, $p<0.05$ ).

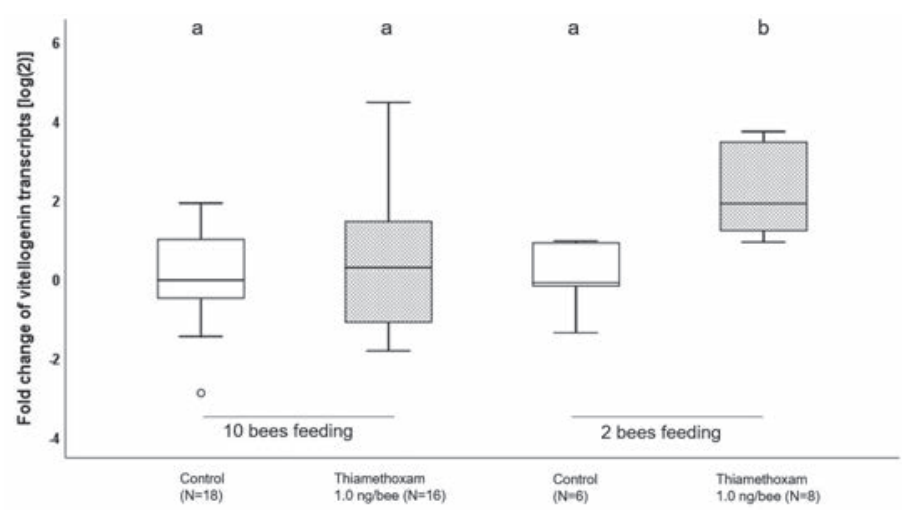

Fig. 6. Change of vitellogenin transcript levels in honey bee brains after oral exposure to $1.0 \mathrm{ng}$ thiamethoxam compared to controls in the feeding scheme of ten bees per group (left) and two bees per group (right) and a consecutive homing flight in the RFID experiment. Letters on top of boxplots mark statistical different groups (Kruskal-Wallis test, two-sided, post-hoc Bonferroni correction for multiple pairwise comparisons, $\mathrm{p}<0.05)$. 
collected by forager bees also has an impact on the filling of their honey stomach: nectar foragers return to their hive with a full honey stomach where they pass the collected nectar to in-hive bees waiting for further processing; a pollen forager's honey stomach, in contrast, is supposed to be emptied during the flight back to the hive. The filling level of the honey stomach of test bees in the RFID homing flight experiments, on the other hand, potentially influences the uptake of contaminated sugar solution. To prevent interferences of syrup uptake with the natural honey stomach content in the RFID test, we therefore strongly suggest to use solely pollen foragers, recognizable on the pollen loads they carry on the outside of their rear legs, rather than mixing pollen and nectar foragers. All pollen foragers should have an empty honey stomach, and using only them in the tests levels the syrup uptake within all test bees, hence makes the treatment dosing more accurate and increases its reliability. Expanding on the second hypothesis that individual treatment of test bees substantiate the reliability of the RFID test by having an equal dosed group of test bees, we adapted the RFID ring test method by using only pollen forager bees and exposing them to sublethal doses of thiamethoxam (1.0 ng per bee and $1.5 \mathrm{ng}$ per bee) and thiacloprid ( $8.0 \mathrm{ng}$ per bee and 250 ng per bee) in a standard group feeding (ten bees per cage), compared to a single bee treatment scheme. The homing ability of thiamethoxam exposed pollen foragers was significantly lower than in untreated controls in the single-bee-feeding approach for both tested doses (Fig. 7; two-sided Welch t-test among all treatment groups and feeding schemes $\mathrm{p}<0.000$, pairwise comparisons with controls and the $1.0 \mathrm{ng}$ and $1.5 \mathrm{ng} / \mathrm{bee}$ treatment groups and posthoc Bonferroni correction for multiple comparisons on arcsine transformed return rates, $\mathrm{p}=0.012$ and $\mathrm{p}=0.006$, respectively), but not in the group-feeding approach (pairwise, two-sided Welch t-tests with Bonferroni correction on arcsine transformed return rates, $\mathrm{p}=1$ and $\mathrm{p}=0.520$ ). Exposure to thiacloprid at the lower dose of $8.0 \mathrm{ng}$ per bee had no effect on the return rate of bees, neither in the group, nor the single-bee feeding regimen (pairwise, two-sided Welch t-tests on arcsine transformed return rates, with Bonferroni correction for pairwise testing, $p>0.05$ ). However, the treatment of bees with $250 \mathrm{ng}$ thiacloprid per bee had, irrespective of the feeding scheme, a strong impact on return rate of bees (Fig. 7; pairwise, two-sided Welch t-tests on arcsine transformed return rates, with Bonferroni correction for pairwise testing, in both feeding schemes $\mathrm{p}<0.001$ ), presumably due to too high dosing, above the sublethal effects limit of thiacloprid.

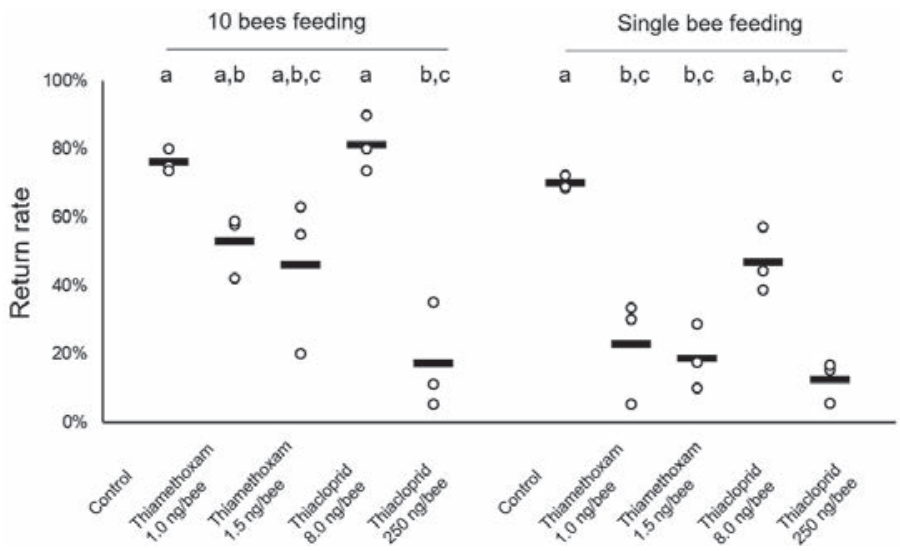

Fig. 7. Return rate of bees in the RFID test exposed to thiamethoxam at doses of 1.0 and $1.5 \mathrm{ng} / \mathrm{bee}$, or to thiacloprid at doses of 8.0 and 250 $\mathrm{ng} / \mathrm{bee}$, either in group feeding scheme (left), or in single feeding (right). Each circle represents the percent of returning bees of a total of 20 bees, in the group feeding scheme bees were confined and treated in cages to ten bees, and in the single-bee feeding scheme 20 bees were caged and treated individually. Letters on top mark statistical differences between groups (Welch-t-test, two-sided, with Bonferroni correction, $p$ $<0.05)$.
Homing time in the single-bee feeding approach was observed to be generally longer in all treatments except for controls than in the group feeding scheme (Fig. 8). We noticed a quicker food uptake in pollen foragers than in a mixed test bee group consisting of nectar and pollen foragers as suggested in the ring test protocol. However, the return rates did not statistically significantly differ when using exclusively pollen foragers (experiment III) compared to a mixed group of foragers (experiment II). Trophallaxis among caged honey bees might therefore have a higher impact on the dose distribution, and in consequence on the return rates in the RFID homing test.

\subsection{Conclusions}

In summary, our results show that the evaluated parameters landscape topography and feeding regime can impact the results of the RFID homing test. A higher variability in return rate and vitellogenin expression was observed in the ten-bee feeding regimen compared to the two- or single-bee feeding regimen. This high variability could probably be caused by inhomogeneous food distribution and in consequence to bias in dosing, e.g. over- or underdosing of individual bees within a replicate of the ten-bee feeding scheme. Single-bee feeding is therefore suspected to facilitate a more accurate and uniform dosage distribution, thus minimizing the potential variability of the collected data. In addition, pollen rather than nectar foragers should be preferred as their honey stomach is generally less full, and they consumed the feed solution faster and more reliably than the nectar foragers. Furthermore, landscape structure had an impact on the return time of the test bees. However, further studies are necessary to analyse this aspect in more details. We also strongly recommend further experiments with different compounds to evaluate the robustness of the RFID method regarding the evaluation of sublethal effects of PPPs on the navigation of foraging bees, including also solitary and bumble bees. Additionally, higher tier study data is needed to translate and understand the extent of the homing failure of single dosed bees at honey bee colony level. Our findings help to improve the RFID homing test method and to reduce possible weaknesses. Unequivocally, validation work on the RFID homing test is necessary and shall continue internationally. An integration of sublethal tests methods and how the resulting data could be appropriately incorporated into the current risk assessment framework needs to be further discussed. The test guidance document on the

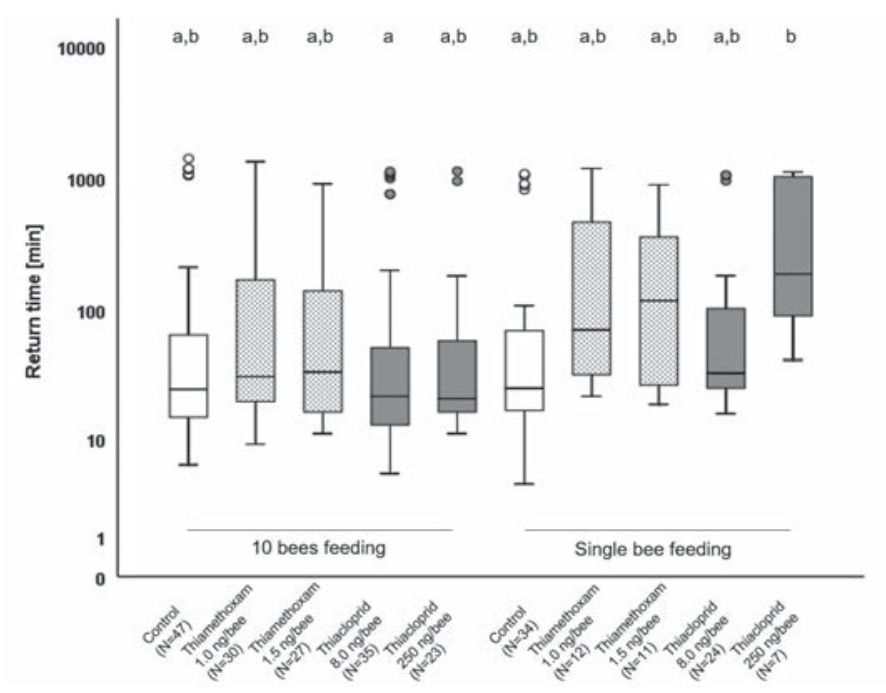

Fig. 8. Return time of bees in the RFID test exposed to thiamethoxam at doses of 1.0 and $1.5 \mathrm{ng} / \mathrm{bee}$, or to thiacloprid at doses of 8.0 and 250 $\mathrm{ng} / \mathrm{bee}$, either in group feeding scheme (left), or in single feeding (right). Letters on top of boxplots mark statistical different groups (KruskalWallis test, post-hoc Bonferroni correction for multiple pairwise comparisons, $p<0.05$ ). 
RFID method is expected to be submitted in 2020 to OECD. The participation in international ring-tests as independent authority aims at learning, co-developing and scrutinizing new methods for the risk assessment of PPPs to bees in respect to PPP authorization, and helps at constantly improving and adjusting the authorization process to the latest knowledge.

\section{Acknowledgements}

We would like to thank Michael Eyer and Pascal Herren for their help in conducting RFID experiments and Benoît Droz for beekeeping. Verena Christen and Tiffany Haltiner provided laboratory assistance and performed gene expression analysis.

Received: December 13, 2019

[1] H. S. Rathore, L. M. Nollet, 'Pesticides: Evaluation of Environmental Pollution', CRC Press, 2012.

[2] United Nations Department of Economic and Social Affairs, Population Division, 'World Population Prospects 2019: Ten Key Findings', United Nations, New York, 2015.

[3] S. Parsa, S. Morse, A. Bonifacio, T. C. Chancellor, B. Condori, V. CrespoPérez, S. L. Hobbs, J. Kroschel, M. N. Ba, F. Rebaudo, Proc. Natl Acad. Sci. 2014, 111, 3889, DOI: 10.1073/pnas.1312693111.

[4] A.-M. Klein, B. E. Vaissiere, J. H. Cane, I. Steffan-Dewenter, S. A Cunningham, C. Kremen, T. Tscharntke, Proc. Roy. Soc. B: Biol. Sci. 2006, 274, 303, DOI: 10.1098/rspb.2006.3721.

[5] M. Albrecht, P. Duelli, C. Müller, D. Kleijn, B. Schmid, J. Appl. Ecol. 2007, 44, 813, DOI: 10.1111/j.1365-2664.2007.01306.x.

[6] a) T. D. Breeze, A. P. Bailey, K. G. Balcombe, S. G. Potts, Agric. Ecosys. Environ. 2011, 142, 137, DOI: 10.1016/j.agee.2011.03.020; b) L. A. Garibaldi, I. Steffan-Dewenter, R. Winfree, M. A. Aizen, R. Bommarco, S. A. Cunningham, C. Kremen, L. G. Carvalheiro, L. D. Harder, O. Afik, Science 2013, 339, 1608, DOI: 10.1126/science.1230200

[7] Der Schweizerische Bundesrat, 'Verordnung 916.161 vom 12. Mai 2010 über das Inverkehrbringen von Pflanzenschutzmitteln (Pflanzenschutzmittelverordnung, PSMV)', as at August 1st 2019.

[8] European Parliament \& Council of the European Union, 'Regulation (EC) No 1107/2009 of the European Parliament and of the Council of 21 October 2009 concerning the placing of plant protection products on the market and repealing Council Directives 79/117/EEC and 91/414/EEC', COD 2006/0136 Official Journal of the European Union, 2009

[9] European Commission, 'Commission Regulation (EU) No 283/2013 of 1 March 2013 setting out the data requirements for active substances, in accordance with Regulation (EC) No 1107/2009 of the European Parliament and of the Council concerning the placing of plant protection products on the market', CELEX: 02013R0283-20141117, EU Publications, 2013.

[10] European Commission, 'Commission Regulation (EU) No 284/2013 of 1 March 2013 setting out the data requirements for plant protection products, in accordance with Regulation (EC) No 1107/2009 of the European Parliament and of the Council concerning the placing of plant protection products on the market', CELEX: 32013R0284, EU Publications, 2013.

[11] V. Storck, D. G. Karpouzas, F. Martin-Laurent, Sci. Total Environ. 2017, 575, 1027, DOI: 10.1016/j.scitotenv.2016.09.167.

[12] a) European and Mediterranean Plant Protection Organization, EPPO Bulletin 2010, 40, 313, DOI: 10.1111/j.1365-2338.2010.02418.x; b)
European and Mediterranean Plant Protection Organization, EPPO Bulletin 2010, 40, 323, DOI: $10.1111 /$ j.1365-2338.2010.02419.x.

[13] European Food Safety Authority, EFSA J. 2013, 11, 268, DOI: 10.2903/j. efsa.2013.3295.

[14] OECD, 'Test No. 213: Honeybees, Acute Oral Toxicity Test', OECD Publishing, Paris, OECD Guidelines for the Testing of Chemicals, Section 2, 1998.

[15] OECD, 'Test No. 214: Honeybees, Acute Contact Toxicity Test', OECD Publishing, Paris, OECD Guidelines for the Testing of Chemicals, Section 2, 1998.

[16] OECD, 'Test No. 245: Honey Bee (Apis Mellifera L.), Chronic Oral Toxicity Test (10-Day Feeding)', OECD Publishing, Paris, OECD Guidelines for the Testing of Chemicals, Section 2, 2017.

[17] OECD, 'Test No. 237: Honey Bee (Apis Mellifera) Larval Toxicity Test, Single Exposure', OECD Publishing, Paris, OECD Guidelines for the Testing of Chemicals, Section 2, 2013.

[18] OECD, 'Guidance Document on Honey Bee Larval Toxicity Test following Repeated Exposure', OECD Publishing, Paris, OECD Series on Testing and Assessment, No. 239, 2016.

[19] P. Oomen, A. De Ruijter, J. Van Der Steen, EPPO Bulletin 1992, 22, 613, DOI: $10.1111 / \mathrm{j} .1365-2338.1992 . t b 00546 . x$.

[20] OECD, 'Guidance Document on the Honey Bee (Apis Mellifera L.) Brood test Under Semi-field Conditions', OECD Publishing, Paris, OECD Series on Testing and Assessment, No. 75, 2014.

[21] OECD, 'Test No. 246: Bumblebee, Acute Contact Toxicity Test', OECD Publishing, Paris, OECD Guidelines for the Testing of Chemicals, Section $2,2017$.

[22] OECD, 'Test No. 247: Bumblebee, Acute Oral Toxicity Test', OECD Publishing, Paris, OECD Guidelines for the Testing of Chemicals, Section 2, 2017.

[23] P. Westrich, 'Wildbienen. Die anderen Bienen', Dr Friedrich Pfeil Verlag, München, 2011.

[24] J. Fourrier, A. Rouzes, C. Monchanin, L. Dubuisson, D. Fortini, P. Aupinel, S. Grateau, C. Vidau, C. Chevallereau, M. Henry, in Conference proceedings of the 14th International Symposium on the hazards of pesticides to bees, International commission for plant-pollinator relationships (ICPP-PR), 2019, p. 32.

[25] A. Decourtye, J. Devillers, P. Aupinel, F. Brun, C. Bagnis, J. Fourrier, M. Gauthier, Ecotoxicol. 2011, 20, 429, DOI: 10.1007/s10646-011-0594-4.

[26] R. Brodschneider, A. Libor, V. Kupelwieser, K. Crailsheim, PloS one 2017, 12, e0174684, DOI: 10.1371/journal.pone.0174684.

[27] V. Christen, F. Mittner, K. Fent, Environ. Sci. Technol. 2016, 50, 4071, DOI: 10.1021/acs.est.6b00678.

[28] M. L. Winston, 'The Biology of the Honey Bee', Harvard University Press, 1991

\section{License and Terms}

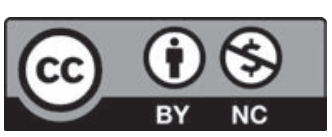

This is an Open Access article under the terms of the Creative Commons Attribution License CC BY_NC 4.0. The material may not be used for commercial purposes.

The license is subject to the CHIMIA terms and conditions: (http://chimia.ch/component/sppagebuilder/?view=page\&id=12).

The definitive version of this article is the electronic one that can be found at doi:10.2533/chimia.2020.176. 